\title{
Os efeitos do exercício físico em pacientes submetidos à hemodiálise: uma revisão sistemática
}

\author{
The effects of physical exercise on patients \\ undergoing hemodialysis: a systematic review
}

\author{
André Rodrigues Carvalho' ${ }^{1}$ \\ Izabelle Macedo de Sousa ${ }^{2}$ \\ Danyele Holanda da Silva ${ }^{3}$ (c) \\ Auriclea Rodrigues da Silva ${ }^{4}$ (C) \\ Rivanda Berenice Silva de Freitas Adad ${ }^{5}$ \\ Vinicius Oliveira da Silva ${ }^{6}$ (1)
}

${ }^{1}$ Autor para correspondência. Centro Universitário Mauricio de Nassau (Teresina). Piauí, Brasil. andre-dez@hotmail.com ${ }^{2-5}$ Centro Universitário Mauricio de Nassau (Teresina). Piauí, Brasil. dan_sil_holanda@hotmail.com, izabelle_macedo@hotmail.com, cleaferdinan@outlook.com,rivandaadad@gmail.com 6Universidade do Vale do Paraíba (São José dos Campos). São Paulo, Brasil. viniciusoliveira.fisio@gmail.com

RESUMO | INTRODUÇÃO: Pacientes portadores de doença renal crônica submetidos à hemodiálise são sedentários devido a dor, fadiga muscular e baixo condicionamento físico. Estudos recentes demonstram os efeitos benéficos de programas de exercícios físicos para estes pacientes durante a hemodiálise resultando em melhor controle da hipertensão arterial, da capacidade funcional, da função cardíaca, da força muscular e, assim, da qualidade de vida. OBJETIVOS: Analisar pesquisas que mostram se o exercício físico produz ou não desfechos clínicos benéficos para indivíduos em hemodiálise. METODOLOGIA: Este artigo constitui-se de uma revisão sistemática composta por ensaios clínicos randomizados publicados entre 2014 e 2019 que relacionassem hemodiálise, exercício físico e qualidade de vida e que apresentassem escore de PEDro superior a 5. Foram excluídos os estudos que estavam em desconformidade com o tema proposto, artigos duplicados entre as bases de dados e intenções de pesquisa. As palavras-chave utilizadas para a busca seguiram a descrição dos termos DECS e MESH, sendo elas: Hemodiálise, exercício físico e qualidade de vida. Essas palavras estavam presentes em qualquer seção do artigo. RESULTADOS: Foram selecionados 15 estudos segundo os critérios de elegibilidade. Os artigos analisados obtiveram um total de 1057 participantes de ambos os sexos com idades entre 18 e 80 anos. Esses foram submetidos a exercícios aeróbicos, anaeróbicos e respiratórios, apresentaram desfechos clínicos benéficos nas três modalidades de exercício físico. CONCLUSÃO: Programas de exercício físico durante a hemodiálise otimizam o ganho da massa, força e resistência muscular, aptidão física, qualidade do sono, autopercepção de saúde, capacidade funcional e consequentemente a qualidade de vida.

\begin{abstract}
INTRODUCTION: Patients with chronic kidney disease submitted to hemodialysis are sedentary due to pain, muscle fatigue and poor physical condition. Recent studies demonstrate the beneficial effects of physical exercise programs to these patients during hemodialysis resulting in better control of hypertension, functional capacity, cardiac function, muscle strength and thus quality of life. OBJECTIVES: To analyze research works that show whether or not exercise produces beneficial clinical outcomes in individuals on hemodialysis. METHODOLOGY: This article is a systematic review of randomized controlled trials published between 2014 and 2019 that related hemodialysis, exercise and quality of life and had a PEDro score $>5$. Studies that were not in conformity with the proposed theme, duplicate articles among the databases and research intentions were excluded. The keywords used for the search followed the description of DECS and MESH terms: Hemodialysis, exercise and quality of life. These words were found in any section of the article. RESULTS: 15 studies were selected according to the eligibility criteria. The articles analyzed had a total of 1057 participants of both sexes aged between 18 and 80 years. They were submitted to aerobic, anaerobic and respiratory exercises, and presented beneficial clinical outcomes in the three modalities of physical exercise. CONCLUSION: Physical exercise programs during hemodialysis optimize mass gain, strength and muscle endurance, physical fitness, quality of sleep, self-perception of health, functional capacity and consequently quality of life.
\end{abstract}

KEYWORDS: Hemodialysis. Physical exercise. Quality of life. 


\section{Introdução}

A insuficiência renal crônica (IRC) é um termo geral para alterações heterogêneas que afetam tanto a estrutura, quanto a função renal, com múltiplas causas e múltiplos fatores de prognóstico 1 . Conforme o censo brasileiro de diálise da Sociedade Brasileira de Nefrologia de $2018^{2}$, mais de 133.000 pacientes realizam terapia renal substitutiva, um aumento de aproximadamente 85.000 pacientes se comparado ao censo de 2002, quando esse número era de 48.000 .

Os fatores etiológicos da IRC envolvem doenças primárias do rim, doenças sistêmicas e doenças hereditárias. As suas causas mais comuns são: diabetes mellitus (DM), glomérulo nefrites, nefroesclerose hipertensiva, doença renovascular, rins policísticos, uropatias obstrutivas e malformações congênitas ${ }^{3}$.

Dentre as diferentes modalidades terapêuticas, a hemodiálise (HD) é a mais utilizada, pois proporciona uma alteração mais rápida na composição de solutos do plasma e na remoção de excesso de água corporal, em comparação com os outros tipos de modalidades de terapia renal substitutiva ${ }^{4}$. A HD, enquanto um procedimento de apoio à função renal, consiste na remoção de substâncias tóxicas e excesso de líquido por uma máquina de diálise, em um procedimento cuja duração leva entre 2 e 4 horas, exigindo que o paciente se desloque para a unidade de tratamento 3 vezes na semana ${ }^{5}$.

O tratamento hemodialítico provoca um cotidiano monótono e restritivo, favorecendo o sedentarismo e a diminuição da capacidade funcional; interferindo negativamente nos contextos emocional e social destes pacientes, que geralmente se sentem fragilizados em função da situação de dependência em que vivem. Estes e outros fatores, como dor, inflamação sistêmica, diminuição da força muscular e mobilidade refletem em uma redução da qualidade de vida6.

Alguns autores ${ }^{7-9}$ indicam que um programa de exercícios físicos durante a diálise promove melhora da capacidade funcional, da força e resistência muscular, da função cardíaca e, consequentemente, da qualidade de vida. Reboredo et al. ${ }^{9}$ defendem que a realização de exercícios durante as sessões de hemodiálise aumenta a aderência ao tratamento, redução da monotonia do processo de diálise e facilidade de acompanhamento médico. Essa revisão sistemática tem por objetivo analisar pesquisas que mostram se o exercício físico produz ou não desfechos clínicos benéficos para indivíduos em HD.

\section{Metodologia}

Constituiu-se de uma revisão sistemática, segundo as diretrizes do protocolo PRISMA. A busca foi realizada nas bases de dados PEDro, PubMed, SciELO e LILACS, através do cruzamento das palavras-chave Hemodiálise, Exercício físico e Qualidade de vida e de seus correspondentes na língua inglesa: Hemodialysis, Exercise e Quality of life. Essas palavras foram retiradas do DeCS e do MESH e estavam presentes em qualquer seção do artigo. A figura 1 representa a estratégia de busca para a base de dados PubMed.

Figura 1. Estratégia de busca para a base de dados PubMed (Setembro, 2019)

- 1 Hemodialysis

- 2 Hemodialysis AND Exercise

- 3 Hemodialysis AND Exercise AND Quality of life

- 4 Hemodialysis AND Exercise AND Quality of life

- 5 Hemodialysis AND Exercise AND Quality of life (filters: Clinical Trial)

- 6 Hemodialysis AND Exercise AND Quality of life (filters: Clinical Trial, 5 years) 
Como critérios de inclusão foram selecionados ensaios clínicos randomizados publicados entre 2014 e 2019 que relacionavam hemodiálise, exercício físico e qualidade de vida e que apresentassem escore de PEDro superior a 5. Critérios de exclusão: desconformidade com o tema proposto, artigos duplicados entre as bases de dados e intenções de pesquisa.

A busca nas bases de dados foi realizada no mês de setembro de 2019. Os artigos foram selecionados inicialmente pela leitura do título e resumo do trabalho, em seguida os estudos selecionados foram analisados por meio da leitura do texto completo a fim de se confirmarem os critérios de elegibilidade.

A qualidade metodológica dos estudos foi avaliada segundo os critérios da escala PEDro ${ }^{10}$, que pontua 11 itens, a saber: 1-Critérios de elegibilidade, 2 - Alocação aleatória, 3 - Alocação oculta, 4 - Comparabilidade da linha de base, 5 - Assuntos cegos, 6 - Terapeutas cegos, 7 - Avaliadores cegos, 8 - Acompanhamento adequado, 9 - Análise de intenção de tratar, 10 -
Comparações entre grupos, 11 - Estimativas pontuais e variabilidade. Os itens são pontuados como presente (1) ou ausente (0), gerando um somatório máximo de 10 pontos, sendo que o primeiro item não é contabilizado.

\section{Resultados}

Na pesquisa inicial foram encontrados 497 artigos (PUBMED: 401; PEDro: 46; LILACS: 29; SCIELO: 21). Após aplicação dos filtros "tipos de artigos", "ano de publicação" e "idioma", restaram 84 estudos potencialmente relevantes para a revisão, dos quais 59 foram excluídos por não estarem de acordo com os critérios de inclusão, resultando em 25 estudos para uma leitura detalha. Após análise dos textos completos, mais 10 estudos foram excluídos, restando 15 artigos incluídos na revisão. O processo de seleção dos estudos está completamente descrito no fluxograma da Figura 2.

Figura 2. Fluxograma de seleção sistemática dos estudos com população submetida a HD, de acordo com a recomendação PRISMA (2019)
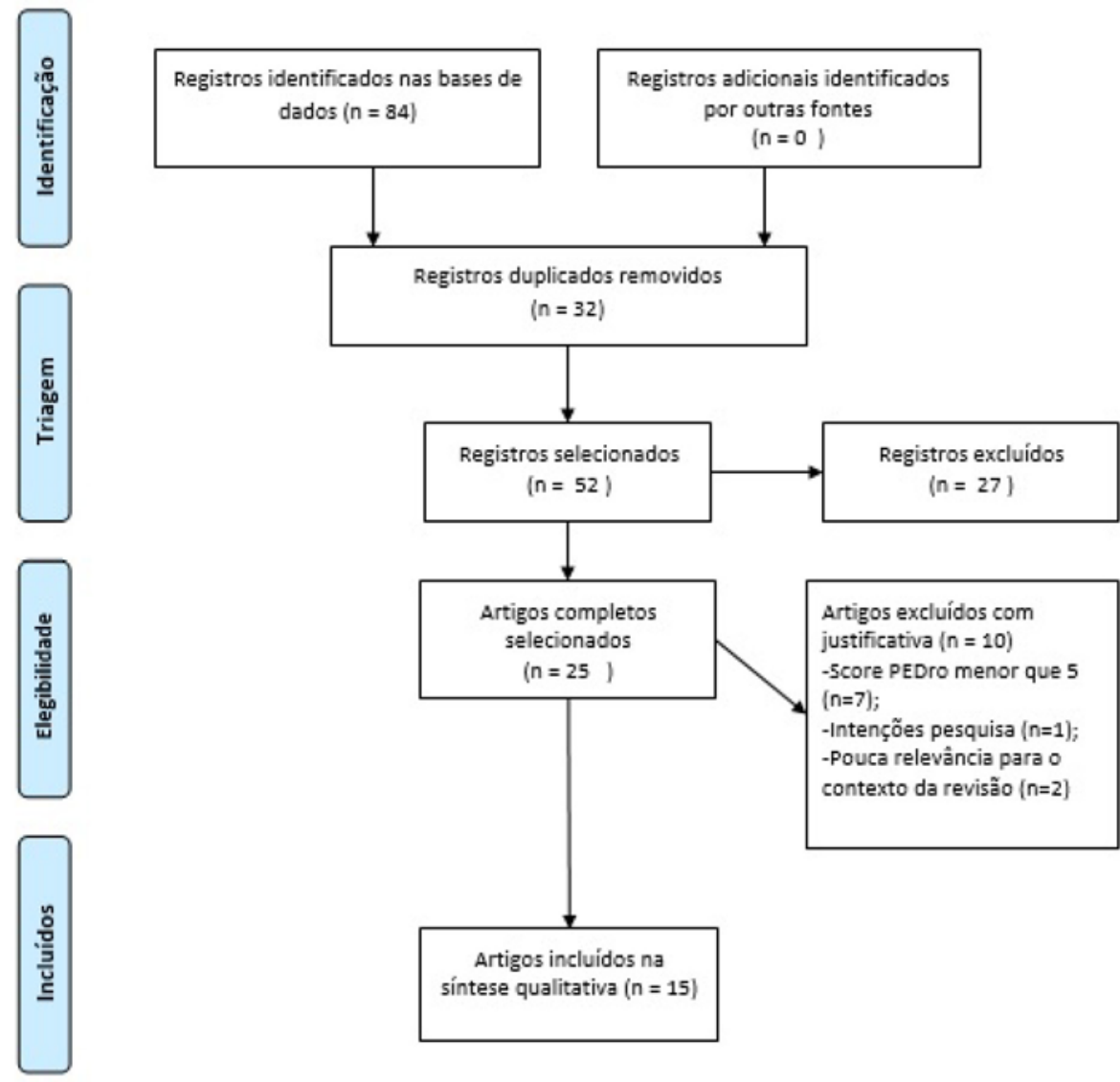
Os artigos selecionados obtiveram um total de 1057 participantes de ambos os sexos com idades entre 18 e 80 anos. Estes foram submetidos a exercícios aeróbicos ${ }^{11,12,15,16,21,23-25}$, anaeróbicos ${ }^{13-15,17-21,23}$ e respiratórios ${ }^{11}$. A caracterização da amostra, os programas de exercícios físicos e os principais desfechos obtidos nos estudos incluídos encontram-se esboçados na Tabela 1, os estudos estão organizados em ordem decrescente de acordo com as pontuações adquiridas no score PEDro.

Tabela 1. Caracterização dos ensaios clínicos randomizados incluídos na revisão sistemática quanto amostra, programas de exercícios físicos e desfechos para população em HD (2019) (continua)

\begin{tabular}{|c|c|c|c|c|c|}
\hline $\begin{array}{l}\text { Autores (Ano } \\
\text { de publicação) }\end{array}$ & $\begin{array}{l}\text { Escore } \\
\text { de } \\
\text { PEDro }\end{array}$ & $\begin{array}{l}\text { Idade } \\
\text { (anos) }\end{array}$ & Amostra & Programa & Desfechos \\
\hline $\begin{array}{l}\text { Figueiredo et } \\
\text { al (2018) }\end{array}$ & 8 & 48,2 & 37 & $\begin{array}{l}\text { G1: TMI a } 50 \% \text { da PImáx. } \\
\text { G2: TA de baixa } \\
\text { intensidade. } \\
\text { G3: TA + TMI. }\end{array}$ & $\begin{array}{l}\text { O TMI e TA combinados } \\
\text { melhoraram os parâmetros } \\
\text { funcionais e biomarcadores } \\
\text { inflamatórios. }\end{array}$ \\
\hline $\begin{array}{l}\text { Tao, Chow, } \\
\text { Wong }(2015)^{12}\end{array}$ & 8 & $27-74$ & 113 & $\begin{array}{l}\text { G1: Exercício resistido + } \\
\text { exercício aeróbio + } \\
\text { gerenciamento dos } \\
\text { exercícios } \\
\text { G2: Exercício resistido + } \\
\text { exercício aeróbio. }\end{array}$ & $\begin{array}{l}\text { O exercício em casa é prático e } \\
\text { eficaz na melhoria da função } \\
\text { física e da autopercepção da } \\
\text { saúde de pacientes em HD. }\end{array}$ \\
\hline $\begin{array}{l}\text { Bennett et al } \\
(2016)^{13}\end{array}$ & 8 & $\begin{array}{l}68.1 \\
(12.6)\end{array}$ & 171 & $\begin{array}{l}\text { Todos os grupos } \\
\text { realizaram exercício físico } \\
\text { resistido progressivo. } \\
\text { G1: } 36 \text { semanas de } \\
\text { intervenção. } \\
\text { G2: } 24 \text { semanas de } \\
\text { intervenção. } \\
\text { G3: } 12 \text { semanas de } \\
\text { intervenção. }\end{array}$ & $\begin{array}{l}\text { O treinamento intradialítico de } \\
\text { resistência pode melhorar a } \\
\text { função física de indivíduos em } \\
\text { HD. }\end{array}$ \\
\hline $\begin{array}{l}\text { Olvera-Soto et } \\
\text { al }(2016)^{14}\end{array}$ & 7 & $21-39$ & 61 & $\begin{array}{l}\text { G1: Exercícios resistidos. } \\
\text { G2: Apenas hemodiálise. }\end{array}$ & $\begin{array}{l}\text { O exercício resistido é seguro e } \\
\text { capaz de melhorar a massa } \\
\text { muscular e força em pacientes } \\
\text { em HD. }\end{array}$ \\
\hline $\begin{array}{l}\text { Marchesan, } \\
\text { Nunes, } \\
\text { Rombaldi } \\
(2014)^{15}\end{array}$ & 7 & $21-40$ & 22 & $\begin{array}{l}\text { G1: Treinamento aeróbio } \\
\text { + exercício resistido. } \\
\text { G2: Apenas hemodiálise. }\end{array}$ & $\begin{array}{l}\text { O treinamento físico melhorou a } \\
\text { aptidão física e a qualidade de } \\
\text { vida dos pacientes em HD. }\end{array}$ \\
\hline $\begin{array}{l}\text { Aoike et al } \\
(2017)^{16}\end{array}$ & 7 & $\begin{array}{l}55,8 \pm \\
8,3\end{array}$ & 40 & $\begin{array}{l}\text { G1: Exercício aeróbio no } \\
\text { centro de HD. } \\
\text { G2: Exercício aeróbio } \\
\text { realizado a domicílio. } \\
\text { G3: Cuidados de rotina } \\
\text { para pacientes em HD. }\end{array}$ & $\begin{array}{l}\text { O treinamento aeróbico a } \\
\text { domicílio foi tão eficaz } \\
\text { como o treinamento no centro } \\
\text { para melhorar o desempenho } \\
\text { físico e } \\
\text { capacidades funcionais, } \\
\text { qualidade de vida e sono nos } \\
\text { pacientes em HD. }\end{array}$ \\
\hline $\begin{array}{l}\text { Rahimimogha } \\
\text { dam, et al } \\
(2017)^{17}\end{array}$ & 6 & $18-65$ & 50 & $\begin{array}{l}\text { G1: Cuidados de rotina } \\
\text { para pacientes em } \\
\text { hemodiálise + exercícios } \\
\text { de Pilates. } \\
\text { G2: Cuidados de rotina } \\
\text { para pacientes em HD. }\end{array}$ & $\begin{array}{l}\text { O Pilates pode ser considerada } \\
\text { uma alternativa eficaz para } \\
\text { melhorar a HD, saúde geral, } \\
\text { estado físico, mental e social dos } \\
\text { pacientes. }\end{array}$ \\
\hline $\begin{array}{l}\text { Suhardjono et } \\
\text { al }(2019)^{21}\end{array}$ & 5 & $\begin{array}{l}48,9 \\
(12,2)\end{array}$ & 120 & $\begin{array}{l}\text { G1: Exercício aeróbio. } \\
\text { G2: Exercício aeróbio + } \\
\text { resistido. } \\
\text { G3: Nenhuma } \\
\text { intervenção. }\end{array}$ & $\begin{array}{l}\text { Ambos os programas de } \\
\text { exercícios aumentaram a força } \\
\text { dos músculos dos MMII } \\
\text { e o índice de qualidade de vida. } \\
\text { O exercício combinado não foi } \\
\text { mais eficaz do que exercício } \\
\text { aeróbico para pacientes em HD. }\end{array}$ \\
\hline
\end{tabular}


Tabela 1. Caracterização dos ensaios clínicos randomizados incluídos na revisão sistemática quanto amostra, programas de exercícios físicos e desfechos para população em HD (2019) (conclusão)

\begin{tabular}{|c|c|c|c|c|c|}
\hline $\begin{array}{l}\text { Autores (Ano } \\
\text { de publicação) }\end{array}$ & $\begin{array}{l}\text { Escore } \\
\text { de } \\
\text { PEDro }\end{array}$ & $\begin{array}{l}\text { Idade } \\
\text { (anos) }\end{array}$ & Amostra & Programa & Desfechos \\
\hline $\begin{array}{l}\text { Lopes et al } \\
(2019)^{18}\end{array}$ & 5 & $30-70$ & 50 & $\begin{array}{l}\text { G1: Treinamento resistido } \\
\text { de alta intensidade. } \\
\text { G1: Treinamento resistido } \\
\text { de intensidade moderada. } \\
\text { G3: Alongamento. }\end{array}$ & $\begin{array}{l}\text { O treinamento resistido de alta } \\
\text { intensidade foi associado a } \\
\text { ganhos de massa magra de MMII } \\
\text { e qualidade de vida, enquanto } \\
\text { capacidade funcional e } \\
\text { sarcopenia foram melhorados } \\
\text { independentemente da } \\
\text { intensidade de treinamento. }\end{array}$ \\
\hline $\begin{array}{l}\text { Rosa et al } \\
(2018)^{20}\end{array}$ & 5 & $\begin{array}{l}55,7 \pm \\
14,03\end{array}$ & 52 & $\begin{array}{l}\text { G1: Treinamento resistido } \\
\text { progressivo. } \\
\text { G2: Mobilização de MMSS } \\
\text { e MMII sem carga e } \\
\text { progressão. }\end{array}$ & $\begin{array}{l}\text { O treinamento resistido } \\
\text { progressivo proporcionou } \\
\text { aumento da massa magra, } \\
\text { resistência, conteúdo mineral } \\
\text { ósseo e flexibilidade em MMII. }\end{array}$ \\
\hline $\begin{array}{l}\text { Tayebi, } \\
\text { Ramezani, } \\
\text { Kashef }(2018)^{19}\end{array}$ & 5 & $18-80$ & 34 & $\begin{array}{l}\text { G1: Exercício resistido } \\
\text { isométrico. } \\
\text { G2: Nenhuma } \\
\text { intervenção. }\end{array}$ & $\begin{array}{l}\text { O exercício isométrico pode } \\
\text { aumentar a capacidade } \\
\text { muscular e ajudar na } \\
\text { manutenção dos níveis de } \\
\text { albumina nos pacientes em HD. }\end{array}$ \\
\hline $\begin{array}{l}\text { Zhao et al } \\
(2017)^{23}\end{array}$ & 5 & $\begin{array}{l}56.9 \pm \\
7.5\end{array}$ & 189 & $\begin{array}{l}\text { G1: Escitalopram } \\
\text { diariamente. } \\
\text { G2: Escitalopram } \\
\text { diariamente + andar de } \\
\text { bicicleta } 6 \text { vezes por } \\
\text { semana. } \\
\text { G3: Medicamento placebo } \\
\text { + andar de bicicleta } 6 \\
\text { vezes por semana. }\end{array}$ & $\begin{array}{l}\text { O exercício aeróbico melhora a } \\
\text { qualidade de vida e melhora a } \\
\text { gravidade da depressão dos } \\
\text { pacientes em hemodiálise, } \\
\text { afetando os níveis de } \\
\text { Interleucina } 6 \text { e } 18 .\end{array}$ \\
\hline $\begin{array}{l}\text { Frih et al } \\
(2017)^{22}\end{array}$ & 5 & $\begin{array}{l}64,7 \pm \\
3,2\end{array}$ & 41 & $\begin{array}{l}\text { G1: Exercício resistido } \\
\text { progressivo + exercício } \\
\text { aeróbio progressivo. } \\
\text { G2: Sem intervenção. }\end{array}$ & $\begin{array}{l}\text { O programa combinado de } \\
\text { treinamento aeróbio e } \\
\text { anaeróbio teve efeito benéfico } \\
\text { na capacidade física e na } \\
\text { qualidade de vida em pacientes } \\
\text { em HD. }\end{array}$ \\
\hline $\begin{array}{l}\text { Matsufuji et al } \\
(2015)^{25}\end{array}$ & 5 & $61-79$ & 17 & $\begin{array}{l}\text { G1: Os pacientes foram } \\
\text { orientados a se levantar e } \\
\text { sentar-se em cadeira de } \\
\text { rodas durante } 15 \\
\text { minutos, com pausas } \\
\text { curtas entre as repetições. } \\
\text { G2: Alongamento } \\
\text { muscular. }\end{array}$ & $\begin{array}{l}\text { O exercício em cadeira de rodas } \\
\text { melhorou as atividades de vida } \\
\text { diária nos pacientes em HD. }\end{array}$ \\
\hline $\begin{array}{l}\text { Bohm et al } \\
(2014)^{24}\end{array}$ & 5 & $\begin{array}{l}53 \pm \\
16.9\end{array}$ & 60 & $\begin{array}{l}\text { G1: Cicloergômetro } \\
\text { durante a hemodiálise. } \\
\text { G1: Exercício aeróbio } \\
\text { gradual baseado no } \\
\text { pedômetro, com meta de } \\
10.000 \text { passos por dia. }\end{array}$ & $\begin{array}{l}\text { O cicloergômetro intradialítico e } \\
\text { o programa de pedômetro em } \\
\text { casa apresentaram melhoras } \\
\text { semelhantes na função e } \\
\text { flexibilidade dos MMII, mas não } \\
\text { alteraram a capacidade aeróbica } \\
\text { dos indivíduos em HD. }\end{array}$ \\
\hline
\end{tabular}

Legenda: G1= Grupo 1; G2= Grupo 2; G3= Grupo 3; MMII= Membros inferiores; MMSS= Membros superiores; $\mathrm{TMI}=$ Treinamento da musculatura inspiratória; TA= Treinamento aeróbico. 
A qualidade de vida foi avaliada em sete estudos ${ }^{15,16,20,22-25}$ por meio da aplicação do questionário SF-36; outros cinco estudos ${ }^{11-13,18,21}$ aplicaram o questionário KDQOL-SF, que avalia a qualidade de vida em doentes renais. A capacidade funcional foi avaliada em nove estudos pelo teste Sit-to-stand (STS) ${ }^{11-16,20,22,25}$ e pelo teste de caminha de 6 minutos (TC-6) em sete $11,15,16,20,22,24,25$.

Com relação a qualidade metodológica dos estudos, dez artigos 11-17,19,21,23 tiveram acompanhamento adequado; seis artigos ${ }^{11-13,15,16,25}$ tiveram alocação oculta e seis ${ }^{11-14,18,22}$ tiveram cegamento dos avaliadores de desfechos. As pontuações do score PEDro de todos os estudos incluídos na revisão estão listados no Quadro 1.

Quadro 1. Pontuações adquiridas no score PEDro dos artigos incluídos na revisão sistemática com população submetida a HD (2019)

\begin{tabular}{|c|c|c|c|c|c|c|c|c|c|c|c|c|}
\hline $\begin{array}{ll}\text { Autores } & \text { Itens (PEDro) } \\
\end{array}$ & 1 & 2 & 3 & 4 & 5 & 6 & 7 & 8 & 9 & 10 & 11 & $\begin{array}{l}\text { Score } \\
\text { Total }\end{array}$ \\
\hline Figueiredo et al. ${ }^{11}$ & 1 & 1 & 1 & 1 & 0 & 0 & 1 & 1 & 1 & 1 & 1 & 8 \\
\hline Tao, Chow, Wong ${ }^{12}$ & 1 & 1 & 1 & 1 & 0 & 0 & 1 & 1 & 1 & 1 & 1 & 8 \\
\hline Bennett et al. ${ }^{13}$ & 1 & 1 & 1 & 1 & 0 & 0 & 1 & 1 & 1 & 1 & 1 & 8 \\
\hline Olvera-Soto et al. ${ }^{14}$ & 1 & 1 & 0 & 1 & 1 & 0 & 1 & 1 & 0 & 1 & 1 & 7 \\
\hline Marchesan, Nunes, Rombaldi ${ }^{15}$ & 1 & 1 & 1 & 1 & 0 & 0 & 0 & 1 & 1 & 1 & 1 & 7 \\
\hline Aoike et al. ${ }^{16}$ & 1 & 1 & 1 & 1 & 0 & 0 & 0 & 1 & 1 & 1 & 1 & 7 \\
\hline Rahimimoghadam, et al. ${ }^{17}$ & 1 & 1 & 0 & 1 & 0 & 0 & 0 & 1 & 1 & 1 & 1 & 6 \\
\hline Suhardjono et al..$^{21}$ & 1 & 1 & 0 & 1 & 0 & 0 & 0 & 1 & 0 & 1 & 1 & 5 \\
\hline Lopes et al. ${ }^{18}$ & 0 & 1 & 0 & 1 & 0 & 0 & 1 & 0 & 0 & 1 & 1 & 5 \\
\hline Rosa et al. ${ }^{20}$ & 1 & 1 & 0 & 1 & 0 & 0 & 0 & 0 & 1 & 1 & 1 & 5 \\
\hline Tayebi, Ramezani, Kashef ${ }^{19}$ & 1 & 1 & 0 & 1 & 0 & 0 & 0 & 1 & 0 & 1 & 1 & 5 \\
\hline Zhao et al. ${ }^{23}$ & 1 & 1 & 0 & 1 & 0 & 0 & 0 & 1 & 0 & 1 & 1 & 5 \\
\hline Frih et al. ${ }^{22}$ & 1 & 1 & 0 & 1 & 0 & 0 & 1 & 0 & 0 & 1 & 1 & 5 \\
\hline Matsufuji et al. ${ }^{25}$ & 1 & 1 & 1 & 1 & 0 & 0 & 0 & 0 & 0 & 1 & 1 & 5 \\
\hline Bohm et al. ${ }^{24}$ & 1 & 1 & 0 & 1 & 0 & 0 & 0 & 0 & 1 & 1 & 1 & 5 \\
\hline
\end{tabular}

\section{Discussão}

Sabe-se que a população em HD tem crescido consideravelmente nos últimos anos. A devida atenção deve ser dada a esses indivíduos, por se tratar de um grupo com alta taxa de mortalidade e graves complicações $^{25}$. Os desfechos dos estudos analisados mostraram o exercício físico como um excelente recurso para melhorar a capacidade funcional, qualidade de vida, autopercepção de saúde e o estado mental e social dos pacientes em HD.

Olvera-Soto et al. ${ }^{14}$ desenvolveram um protocolo para pacientes em HD com inclusão de exercícios físicos resistidos com faixas elásticas e tornozeleiras de peso durante um período de 12 semanas. Neste estudo os autores comprovaram a relevância da inserção de exercícios físicos resistidos durante a hemodiálise para melhorar a massa e a força muscular. Contudo, vale ressaltar que otimizar a força não necessariamente implica em melhorar a função física.
Bennett et al. ${ }^{13}$ em seu estudo observaram um declínio na força e função física dos pacientes que estavam há 3, 6 e 9 meses em HD sem realizar qualquer tipo de exercício físico. Após um programa de exercícios resistidos foi constatado uma melhora tanto na força muscular como na função física destes indivíduos.

Bohm et al. ${ }^{24}$ propuseram dois protocolos de intervenções baseados em exercícios aeróbios com cicloergômetro e com utilização do pedômetro e verificaram que as duas modalidades foram capazes de melhorar a função física dos pacientes em HD. Resultados semelhantes a estes foram encontrados por Marchesan, Nunes, Rombaldi ${ }^{15}$ ao aplicar um protocolo de exercício físico que incluía treinamento aeróbico em cicloergômetro e treinamento resistido progressivo.

Em oposição a estes resultados, um protocolo de exercício resistido proposto por Rosa et al. ${ }^{22}$ foi ineficaz para melhorar a capacidade funcional e qualidade de vida dos indivíduos em HD. Porém, os resultados desfavoráveis podem ter se dado pelo fato do 
grupo controle ter realizado exercício simulado o que pode ter influenciado um potencial efeito placebo sobre esse grupo. Além disso o estudo teve algumas limitações, como alocação não secreta e não cegamento dos terapeutas, avaliadores e participantes, o que também pode ter interferido nos resultados.

Figueiredo et al. ${ }^{11}$ observaram em seu estudo que o grupo submetido a treinamento da musculatura inspiratória teve uma melhora significativa nos parâmetros funcionais e nos níveis da proteína C-reativa, um biomarcador de inflamação crônica. Outros dois importantes biomarcadores de inflamação são a IL6 e IL18. Zhao et al. ${ }^{23}$ observaram que os participantes que andaram de bicicleta por 1 hora durante 18 semanas com frequência de 6 vezes por semana, tiveram redução dos níveis séricos desses dois biomarcadores.

Em contrapartida, o protocolo de exercício aeróbio proposto por Suhardjono et al. ${ }^{21}$ não alterou os níveis dos biomarcadores de inflamação avaliados. Entretanto, os autores ressaltam que o protocolo de exercício pode não ter sido adequadamente eficaz e que os níveis dos biomarcadores nos participantes tiveram uma ampla variação, o que dificultou a análise entre grupos.

Aoike et al. ${ }^{16}$ e Tao, Chow, Wong ${ }^{12}$ investigaram os efeitos de um programa de exercício físico realizado a domicílio sob supervisão, em comparação ao treinamento realizado nos centros de HD. Os dois estudos concluíram que um programa de exercício físico realizado em domicílio sob supervisão é tão eficaz na melhora da capacidade funcional e qualidade de vida quanto o realizado nos centros de HD, mostrando que o exercício domiciliar pode ser um método capaz de garantir o envolvimento destes pacientes em programas de treinamento físico.

Apesar dos benefícios do exercício físico para a população em HD já estarem bem elucidados na literatura, a implementação de programas de atividade física nos centros de hemodiálise ainda é um desafio, sejam por barreiras levantadas pelos próprios pacientes ou por restrições de recursos. Dessa forma, se fazem necessárias estratégias capazes de garantir o envolvimento desta população em programas de treinamento físico.

O estudo apresenta algumas limitações: a variabilidade metodológica e de desfechos dos artigos analisados interferiram na comparação dos resultados obtidos na pesquisa, principalmente com relação aos programas de exercícios físicos que variaram quanto ao tempo de acompanhamento, intensidade do treinamento e o tipo de intervenção aplicada. O treinamento muscular inspiratório foi utilizado em apenas um estudo e este foi realizado em associação ao treinamento aeróbico, desta forma não se pode confirmar os reais benefícios dessa modalidade de intervenção na população em HD. Além disso, nenhum dos estudos realizaram as intervenções com cegamento dos participantes e terapeutas o que interfere na qualidade metodológica dos mesmos.

\section{Conclusão}

Programas de exercício físico durante a HD produzem desfechos clínicos benéficos. Estes otimizam o ganho da massa, força e resistência muscular, aptidão física, qualidade do sono, autopercepção de saúde, capacidade funcional e consequentemente a qualidade de vida.

\section{Contribuição dos autores}

Todos os autores contribuíram igualmente para o desenvolvimento do estudo.

\section{Conflitos de interesses}

Nenhum conflito financeiro, legal ou político envolvendo terceiros (governo, empresas e fundações privadas, etc.) foi declarado para nenhum aspecto do trabalho submetido (incluindo, mas não se limitando a subvenções e financiamentos, participação em conselho consultivo, desenho de estudo, preparação de manuscrito, análise estatística, etc.).

\section{Referências}

1. Ministério da Saúde. Diretrizes clínicas para o cuidado ao paciente com doença renal crônica - DRC no Sistema Único de Saúde. Brasília: Ministério da Saúde; 2014.

2. Sociedade Brasileira de Nefrologia. Censo de Diálise SBN. [Internet]. 2018. [acesso em 2019 set. 16]. Disponível em: https:// sbn.org.br/o-censo-2018-foi-publicado-confira/

3. Bianchi, PD. Avaliação da Função Pulmonar e estresse oxidativo em indivíduos com Insuficiência Renal Crônica em Hemodiálise [dissertação]. Porto Alegre: Universidade Federal do Rio Grande do Sul; 2003. 
4. Terra FS, Costa AMDD, Figueiredo ET, Morais AM, Costa $\mathrm{MD}$, Costa RD. As principais complicações apresentadas pelos pacientes renais crônicos durante as sessões de hemodiálise. Rev Bras Clin Med. 2010;8(3):187-92.

5. Thomas CV, Alchieri JC. Qualidade de vida, depressão e características de personalidade em pacientes submetidos à hemodiálise. Avaliação Psicológica. 2005;4(1):57-64.

6. Frazão CMFQ, Ramos VP, Lira ALBC. Qualidade de vida de pacientes submetidos a hemodiálise. Rev Enferm. 2011;19(4):57782.

7. Peres CPA, Kovelis D, Arnaut AC, Bonomo C, Ferrari R, Santos GCCO et al. Efeitos de um programa de exercícios físicos em pacientes com insuficiência renal crônica em hemodiálise. $13^{\circ}$ Simpósio Internacional de Fisioterapia Respiratória e Fisioterapia em Terapia Intensiva, 2006; Curitiba, Paraná: Associação Brasileira de Pesquisa e Pós-Graduação em Fisioterapia; 2006. p.6-9.

8. Reboredo MM, Henrique DMN, Bastos MG, Paula RB. Exercício físico em pacientes dialisados. Rev Bras Med Esporte. 2007;13(6):427-30. doi: 10.1590/S1517-86922007000600014

9. Reboredo MM, Faria RS, Portes LH, Mol CG, Pinheiro BV, de Paula RB. Exercício aeróbico durante a hemodiálise: relato de cinco anos de experiência. Fisioter. Mov. 2011; 24(2): 239-246. doi: $\underline{10.1590 / 50103-51502011000200005}$

10. Morton NA. The PEDro scale is a valid measure of the methodological quality of clinical trials: a demographic study. Aust J Physiother. 2009;55(2):129-133. doi: 10.1016/S00049514(09)70043-1

11. Figueiredo PHS, Lima MMO, Costa HS, Martins JB, Flecha $\mathrm{OD}$, Gonçalves PF et al. Effects of the inspiratory muscle training and aerobic training on respiratory and functional parameters, inflammatory biomarkers, redox status and quality of life in hemodialysis patients: a randomized clinical trial. PLoS One. 2018;13(7):e0200727. doi: 10.1371/journal.pone.0200727

12. Tao $X$, Chow SKY, Wong FKY. A nurse-led case management program on home exercise training for hemodialysis patients: $A$ randomized controlled trial. Int J Nurs Stud. 2015;52(6):1029-41. doi: 10.1016/j.ijnurstu.2015.03.013

13. Bennett PN, Fraser S, Barnard R, Haines T, Ockerby C, Street $M$ et al. Effects of an intradialytic resistance training programme on physical function: a prospective stepped-wedge randomized controlled trial. Nephrol Dial Transplant. 2016;31(8):1302-9. doi: 10.1093/ndt/gfv416

14. Olvera-Soto MG, Valdez-Ortiz R, López AJC, Espinosa-Cuevas ML. Effect of resistance exercises on the indicators of muscle reserves and handgrip strength in adult patients on hemodialysis. J Ren Nutr. 2016;26(1):53-60. doi: 10.1053/j.jrn.2015.06.006

15. Marchesan M, Nunes VGS, Rombaldi AJ. Physical training improves physical fitness and the quality of life of patients on hemodialysis. Rev Bras Cineantropom Desempenho Hum. 2014;16(3). doi: 10.5007/1980-0037.2014v16n3p334
16. Aoike DT, Baria F, Kamimura MA, Ammirati A, Cuppari L. Home-based versus center-based aerobic exercise on cardiopulmonary performance, physical function, quality of life and quality of sleep of overweight patients with chronic kidney disease. Clin Exp Nephrol. 2018;22(1):87-98. doi: 10.1007/s10157017-1429-2

17. Rahimimoghadam Z, Rahemi Z, Mirbagher Ajorpaz N, Sadat $Z$. Effects of Pilates exercise on general health of hemodialysis patients. J Bodyw Mov Ther. 2017;21(1):86-92. doi: 10.1016/j. jbmt.2016.05.012

18. Lopes LCC, Mota JF, Prestes J, Schincaglia RM, Silva DM, Queiroz NP. Intradialytic resistance training improves functional capacity and lean mass gain in hemodialysis patients: a randomized pilot trial. Arch Phys Med Rehabil. 2019;100(11):21512158. doi: 10.1016/j.apmr.2019.06.006

19. Tayebi M, Ramezani A, Kashef M. The Effect of Intradialytic Isometric Resistance Training on Muscle capacity and Serum Albumin Levels in Hemodialysis Patients. Nephro-Urol Mon. 2018;10(2):e65081. doi: 10.5812/numonthly.65081

20. Rosa CSDC, Nishimoto DY, Souza GDE, Ramirez AP, Carletti $C O$, Daibem CGL et al. Effect of continuous progressive resistance training during hemodialysis on body composition, physical function and quality of life in end-stage renal disease patients: a randomized controlled trial. Clin Rehabil. 2018;32(7):899-908. doi: 10.1177/0269215518760696

21. Suhardjono, Umami V, Tedjasukmana D, Setiati S. The effect of intradialytic exercise twice a week on the physical capacity, inflammation, and nutritional status of dialysis patients: a randomized controlled trial. Hemodial Int. 2019;23(4):486-493. doi: $10.1111 /$ hdi.12764

22. Frih B, Jaafar H, Mkacher W, Ben Salah Z, Hammami M, Frih A. The Effect of Interdialytic Combined Resistance and Aerobic Exercise Training on Health Related Outcomes in Chronic Hemodialysis Patients: The Tunisian Randomized Controlled. Front Physiol. 2017;8:288. doi: 10.3389/fphys.2017.00288

23. Zhao C, Ma H, Yang L, Xiao Y. Long-term bicycle riding ameliorates the depression of the patients undergoing hemodialysis by affecting the levels of interleukin- 6 and interleukin-18. Neuropsychiatr Dis Treat. 2016;13:91-100. doi: 10.2147/NDT.S124630

24. Bohm C, Stewart K, Onyskie-Marcus J, Esliger D, Kriellaars D, Rigatto C. Effects of intradialytic cycling compared with pedometer on physical function in chronic outpatient hemodialysis: a prospective randomized trial. Nephrol Dial Transplant. 2014;29(10):1947-55. doi: 10.1093/ndt/gfu248

25. Matsufuji S, Shoji T, Yano Y, Tsujimoto Y, Kishimoto H, Tabata T et al. Effect of Chair Stand Exercise on Activity of Daily Living: A Randomized Controlled Trial in Hemodialysis Patients. J Ren Nutr. 2015;25(1):17-24. doi: 10.1053/j.jrn.2014.06.010 